\title{
Properties and Performance of Two Wide Field of View Cherenkov/Fluorescence Telescope Array Prototypes
}

\author{
S. S. Zhang ${ }^{\mathrm{a}, *}$, Y. X. Bai ${ }^{\mathrm{a}}$, Z. Cao ${ }^{\mathrm{a}}$, S. Z. Chen ${ }^{\mathrm{a}}$, M. J. Chen ${ }^{\mathrm{a}}$, Y. Chen ${ }^{\mathrm{a}}$, L. H. Chen ${ }^{\mathrm{b}}$, K. Q. Ding ${ }^{\mathrm{a}}$, H. H. He ${ }^{\mathrm{a}}$, J. L. \\ Liu $^{\mathrm{a}}$, X. X. Li ${ }^{\mathrm{a}}$, J. Liu ${ }^{\mathrm{a}}$, L. L. Ma ${ }^{\mathrm{a}}$, X. H. Ma ${ }^{\mathrm{a}}$, X. D. Sheng ${ }^{\mathrm{a}}$, B. Zhou ${ }^{\mathrm{a}}$, Y. Zhang ${ }^{\mathrm{a}}$, J. Zhao ${ }^{\mathrm{a}}$, M. Zha $^{\mathrm{a}}$, G. Xiao $^{\mathrm{a}}$ \\ ${ }^{a}$ Institute of High Energy Physics, CAS, Beijing 100049. \\ ${ }^{b}$ Heibei Normal University, China, Heibei 050016.
}

\begin{abstract}
A wide field of view Cherenkov/fluorescence telescope array is one of the main components of the Large High Altitude Air Shower Observatory project. To serve as Cherenkov and fluorescence detectors, a flexible and mobile design is adopted for easy reconfiguring of the telescope array. Two prototype telescopes have been constructed and successfully run at the site of the ARGO-YBJ experiment in Tibet. The features and performance of the telescopes are presented.
\end{abstract}

Keywords: WFCTA, Cherenkov telescope, fluorescence telescope, Cosmic ray detector.

\section{Introduction}

The energy spectrum of primary cosmic rays spans almost 12 orders of magnitude, from $10^{9} \mathrm{eV}$ to $10^{21} \mathrm{eV}$, and can be well fitted by a simple power law except in several small energy regions. A region called the "knee" of the spectrum existing at around $10^{15} \mathrm{eV}$ is one of these regions where the spectrum becomes steeper at higher energy side. Many experiments have observed this phenomenon; however, controversial arguments on its origin persist because of limited discrimination power on the primary cosmic ray composition and ambiguities in nucleus-nucleus interaction modeling. These two aspects are closely related to each other. Modern balloon borne experiments, such as ATIC [1] and CREAM [2], have efficiently measured the energy spectra of individual elements at the top of the atmosphere. The energy spectra for all nuclei are measured up to $\sim 100 \mathrm{TeV}$ which is not far from the "knee". Because the detector area is constrained by the payload, the spectrum measurement has to be extended to a higher energy using a ground based air shower detector array. The spectrum should initially be measured well below $100 \mathrm{TeV}$ to create an overlap with the balloon experiments which serve as absolute calibrations

${ }^{*}$ Corresponding author. Institute of High Energy Physics, CAS, Beijing 100049. Tel.: +86-010-88236035

Email address: zhangss@ihep.ac.cn (S. S. Zhang) for the ground-based techniques. Identifying the individual components of cosmic rays continues to be a major challenge in ground-based experiments. Multiple parameter measurements on an air shower seem to be a plausible approach. The ultimate goal is to separate individual species out of the total observedevent samples and measure a clear individual "knee" for every single species, enabling the discovery of the origin of the "knee". As one of the major scientific goals of the Large High Altitude Air Shower Observatory (LHAASO) project [3, 4], the energy spectrum for a separated composition will be measured at energies above dozens of $\mathrm{TeV}$. To tag each primary particle that causes an air shower, the atmospheric depth of the shower maximum should be measured as one of the important parameters. The wide field of view Cherenkov/fluorescence telescope array (WFCTA), one of the components of the LHAASO project, is designed to accomplish this goal.

A portable design of WFCTA telescopes is adopted to maximize the flexibility of changing the configuration of the array of telescopes. The elevations, pointing directions, and locations of the telescopes are then easily reconfigured. This is one way of using the same telescopes to serve as both fluorescence and Cherenkov detectors. In the fluorescence detector, the telescopes are tilted down to a horizontal position. In such an operational mode, which is analogous to the HiRes experiment [13], most of the Cherenkov photons are 
avoided except those that are scattered onto the field 104 of views (FOVs) of the telescope, such as in the fluo- 105 rescence detector of the telescope array experiment [10] 106 and the fluorescence detector of the Pierre Auger experi- 107 ment [8, 9]. Only the fluorescence light from the shower 108 is collected together with the scattered Cherenkov light 109 to trigger the telescopes. This requires showers hav- 110 ing much higher energy, usually above $100 \mathrm{PeV}$, such 111 as in the HiRes prototype experiment [15], because 112 the fluorescence light by a single electron is consid- 113 erably weaker and isotropic. In the Cherenkov detec- 114 tor, the telescopes run in high elevation mode to di- 115 rectly measure Cherenkov light from the showers, sim- 116 ilar to what was done in the Dice experiment [16]. A ${ }_{117}$ Cherenkov light radiation provides considerably more 118 photons along the shower axis that are useful for lower- ${ }_{119}$ ing the shower energy.

In 2007, two prototype Cherenkov telescopes [5, 6] ${ }_{121}$ were deployed at Yangbaijing (YBJ) Cosmic Ray Ob- 122 servatory near the ARGO-YBJ experiment [7]. More- 123 over, two WFCTA telescopes have been successfully 124 running in Cherenkov mode beginning August 2008. To 125 date, millions of cosmic ray events that simultaneously 126 trigger the telescopes and the ARGO-YBJ detector car- 127 pet array have been collected. An analysis of these 128 events is carried out to study the performance of the tele- ${ }_{129}$ scopes. Detailed descriptions of the telescopes and the 130 analysis of the findings are presented in this paper. $\quad{ }_{131}$

Several details about the apparatus are presented in ${ }_{132}$ Section 2. The detector calibration is then discussed in ${ }_{133}$ Section 3. The test-run of the two telescopes and re- 134 sults are reported in Section 4 including summaries on 135 the detector performance. The conclusions drawn are 136 provided in the last section.

\section{Apparatus}

The two prototype telescopes are deployed near the ${ }_{141}$ ARGO-YBJ carpet detector array at a longitude of 142 $90.53^{\circ} \mathrm{E}$, and a latitude of $30.11^{\circ} \mathrm{N}$ and $4300 \mathrm{~m}$ a.s.l. ${ }_{143}$ One telescope is about $25 \mathrm{~m}$ away from the west side 144 of the ARGO-YBJ array. The other is also $25 \mathrm{~m}$ away 145 from the south side of the array with separation distance between the two telescopes is $50 \mathrm{~m}$. Each telescope has 146 an FOV of $14^{\circ}$ in elevation by $16^{\circ}$ in azimuth. The fo- ${ }_{147}$ cal plane camera is made of a $16 \times 16$ photomultiplier 148 tube (PMT) array, and the pixel size is approximately 149 $1^{\circ}$. Because both telescopes are tilted up to $60^{\circ}$ pointing 150 in the same direction, they can be operated in stereo- 151 scopic mode, i.e., showers striking an area covered by 152 the telescopes will be seen simultaneously. Since the 153 Cherenkov light from a shower is very concentrated in a 154 forward region; thus, the telescopes can be triggered by showers coming within a cone of approximately $8^{\circ}$ with respect to the main optic axes of the telescopes.

The entire telescope system is composed of an optic ultraviolet light collector, a focal plane camera, front end electronics (FEE) based on $50-\mathrm{MHz}$ flash analogto-digital-converters (FADC), data acquisition (DAQ) based on an embedded ARM processor and PC104 bus, power supplies for low and high voltages, and a slow control system. Everything is installed in a shipping container with dimensions of $2.5 \mathrm{~m} \times 2.3 \mathrm{~m} \times 3 \mathrm{~m}$ (Fig 1). Mirrors are mounted at one end of the container and the camera is located at the other end where the focal planes of the mirrors are. The FEE and DAQ are placed at the back plane of the PMT camera. A glass window is installed at the entrance aperture to keep dust from entering the apparatus. The container is mounted on a dump-truck frame with a hydraulic lift that allows the container to be lifted up from $0^{\circ}$ to $60^{\circ}$. The mobility of the entire telescope allows for freely switch between configurations of the telescope array for different observational modes. The architecture of the electronic data acquisition and the slow control system are shown in Fig, 2, whereas that of a sub-cluster is shown in Fig 3 . The PMT signals are processed using an analog processing board (AB) and then a digitization board (DB). The first level trigger (FLT) is generated in the DB on a subcluster. After the FLT is determined, 256 FLTs are then sent to the trigger board (TB). The second level trigger (SLT) and the third level trigger (TLT) are then determined in the TB. After this, the event trigger from the TB is fanned out by a bus driver board (BDB) and sent back to each of the DB and GPS boards. The data are initially stored in the buffer of the DB when the DB has received the event trigger, after which the data that include the GPS time are read using TS7200. Finally, the data are stored in a PC in the laboratory via Ethernet. A detailed description of the detector, divided into the following 8 subsystems, is provided: 1) optics, 2) camera, 3) FEE, 4) trigger system, 5) DAQ, 6) power supply system, 7) slow control system that includes monitoring of everything, and 8) calibration.

\subsection{Optics}

A $4.7 \mathrm{~m}^{2}$ spherical aluminized mirror, composed of 20 hexagon-shaped segments, is used as an ultraviolet light collector. Each segment is subjected to a strict control of the surface quality and their geometrical and optical properties. The reflectivity is greater than $82 \%$ for light having a wavelength $\geq 300 \mathrm{~nm}$. The radius of the curvature of the segments is $4740 \mathrm{~mm}$ with a tolerance of $\pm 20 \mathrm{~mm}$. 


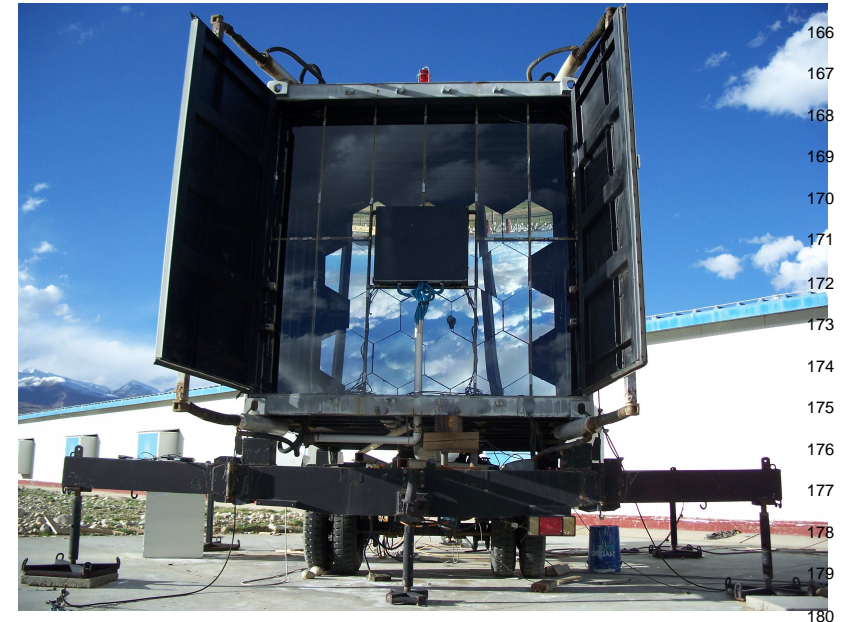

Figure 1: Photograph of the telescope with the doors open.

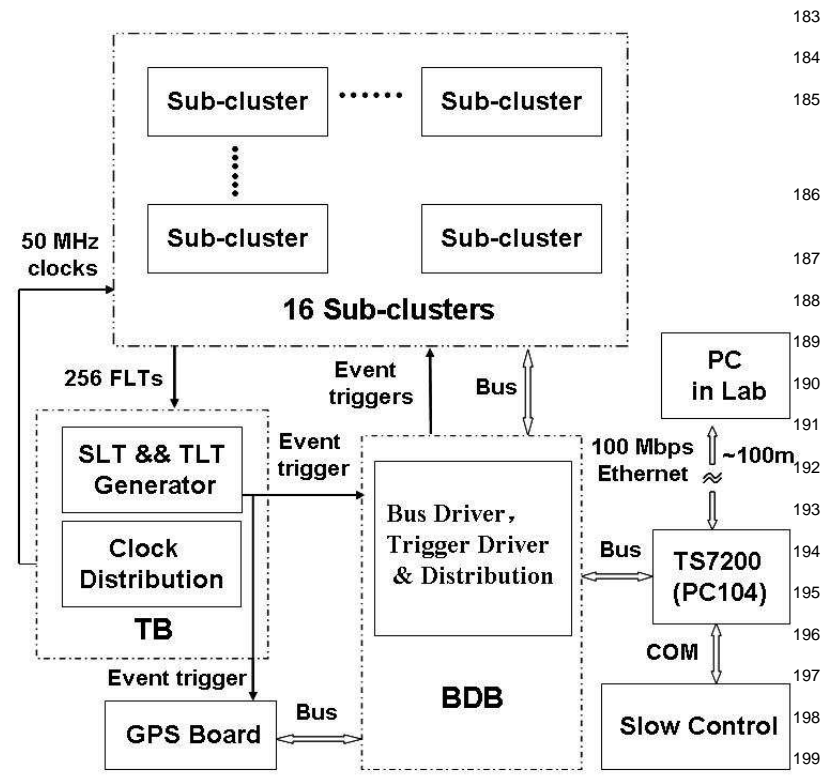

Figure 2: Communications diagram of one telescope; for details of 201 the sub-cluster see Fig 3

The size of a light spot on the focal plane where the 205 PMT camera is located is designed to be similar to the 206 size of a pixel. The sensitivity of a PMT is non-uniform 207 across the surface of the photocathode [17], and small 208 gaps exist between PMTs in the camera. For a spot 209 that is exceedingly larger than the dimension of the gaps 210 and the typical spatial scale of the non-uniformity across 211 the photocathode, light from a specific direction will be 212 shared by a few adjacent PMTs. Thus, effects stemming 213 from the overall non-uniformity of response across the 214 entire camera are reduced. The direction of incident 215 photons can be efficiently determined by simply taking the average of the directions of the registered pixels as weighted by the measured charge in a pixel. Contrastingly, the size of a spot is optimized to be similar to pixel size to avoid sharing of incident photons by an excessive number of pixels.

Because of the abbreviation of the spherical reflector, the spot size changes across the focal plane in a rather large FOV. Large comas also occur at large off-axis angles. The uniformity of the spot size over the camera is also optimized by locating the camera slightly away from the focus. A distance of $2305 \mathrm{~mm}$ between the mirror and the camera is eventually set according to a detailed ray-tracing calculation.

Each mirror segment is mounted on a spherical steel frame with three adjustable screws. The pointing orientations of all segments are adjusted toward the geometric center of the curvature. Using a laser beam, the orientations are calibrated to be less than 7.6 arc seconds from the nominal direction.

\subsection{PMT Camera}

Photons in a spot at the focal plane are recorded by a camera composed of 256 pixels which are $40 \mathrm{~mm}$ Photonis hexagonal PMTs (XP3062/FL). The camera is enclosed in a box and mounted on a frame at a distance of $2305 \mathrm{~mm}$ from the spherical mirror.

The PMTs in a telescope are operated at a gain of $6 \times 10^{5}$. To achieve the best uniformity at hardware level, a resistor is placed between the high voltage power supply and each PMT base to compensate for the gain difference between PMTs. The gains $(\mathrm{G})$ for all PMTs and their responses to the supplied voltage, i.e., $G \propto V^{-\beta}$, are calibrated. All $\beta$ values are measured and recorded in a database in the laboratory for future use. The PMTs are then sorted according to their gains and grouped into two classes. For instance, the working voltages of the PMTs of one of the telescopes distribute $1088 \mathrm{~V}$ to the telescope in a common power supply with a variance of $73.97 \mathrm{~V}$. The average $\beta$ of these tubes is 5.9 with a variance of 0.6 .

The 16 PMTs, as an integrated unit, are soldered on a high voltage board (HVB) that distributes a negative high voltage to all cathodes and dynodes of the PMTs. The voltage division scheme is recommended by Photonis to yield the maximum gain of the tubes. The maximum output current of the PMTs allows a range of 3.5 orders of magnitude in which the non-linearity of all tubes is less than $8 \%$ according to a calibration in the laboratory [17]. The anode signals are finally DC coupled to the FEE. 

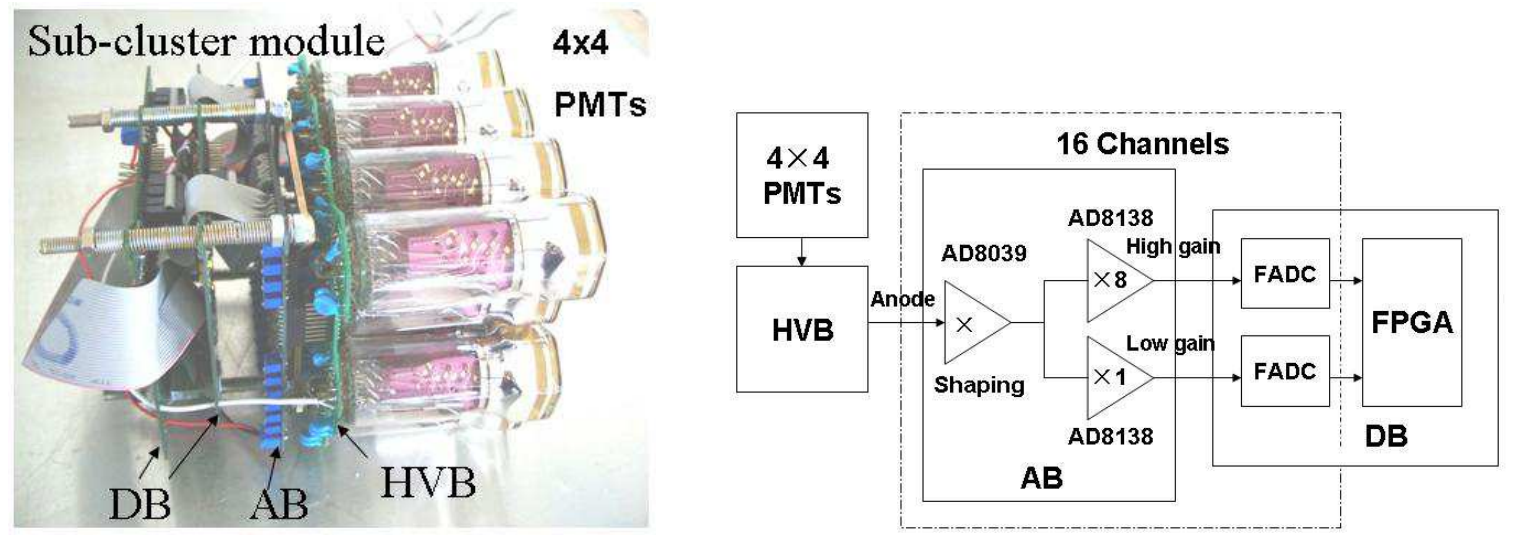

Figure 3: Photograph of a sub-cluster (left) and schematic of the sub-cluster (right).

\subsection{FEE and Digitization}

The FEE is located behind the HVB, which is composed of an $\mathrm{AB}$ and two DBs, to avoid a long distance transmission of the analog signals. Such a module is referred to as a sub-cluster (see the left figure of Fig 3 ) in this paper. A block diagram on the procession of signals from the PMTs to the on-board data storage chip is shown in the right figure of Fig 3 The signal of each PMT is transmitted to the first amplifier on the $\mathrm{AB}$ for noise filtering and pulse stretching. Upon division into high or low gain channels, the signal is then sent to the DB for digitization and further processing. The rest of this section describes the processing procedure in detail.

\subsubsection{Analog Processing Board}

Each $\mathrm{AB}$ has 16 channels for the signals from 16 PMTs on HVB alone. Each channel has a shaping circuit and amplifiers for low or high gains. The board has four main functions, namely:

- low-pass filter of $20 \mathrm{MHz}$,

- expanding narrow pulses,

- dual-gain system for covering a dynamic range over 3.5 orders of magnitude,

- and receiving PMT signals from HVB and performing a single-ended-to-differential conversion.

The anode signals are fed to a four-pole low-pass 259 filter based on an AD8039 through DC coupling. 260 Cherenkov photons generated by all shower particles 261 move at almost the same speed as the charged particles; 262 thus, all photons generated over the entire shower de- 263 velopment beginning from the top of the atmosphere hit 264 the cathodes at almost the same time. The duration of 265

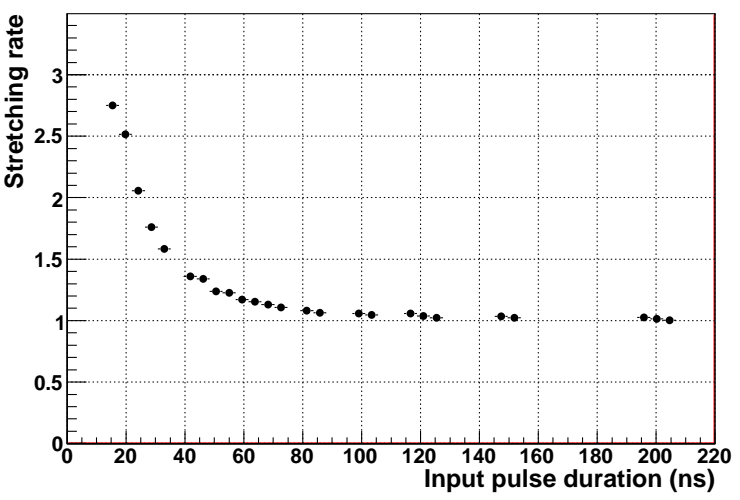

Figure 4: Stretching ratio as a function of the input pulse duration

the pulse lasts only a few nanoseconds, which is shorter than the typical response time of the PMT, e.g., a pulse duration generated by a single-photo-electron (SPE) is approximately $12 \mathrm{~ns}$ on average. Therefore, such a narrow pulse has to be stretched (e.g., at $>50 \mathrm{~ns}$ ) to be able to measure the charge at a sampling rate of 50 $\mathrm{MHz}$, as preselected for the FADCs on DBs. Taking into account the optimized bandwidth of the noise filter, the stretching ratio is selected in such a manner that the narrower pulses are stretched further, and pulses wider than 120 ns are essentially not stretched. In Fig 4 the stretching ratio is plotted as a function of the pulse duration. According to this, the original waveform is reconstructible, and the timing of the pulse can be corrected with an uncertainty less than the duration of the stretched pulses.

The amplification of the PMT signals with a gain of 2.67 is another feature of the shaping circuit. The value of the gain is also optimized together with the filter and 


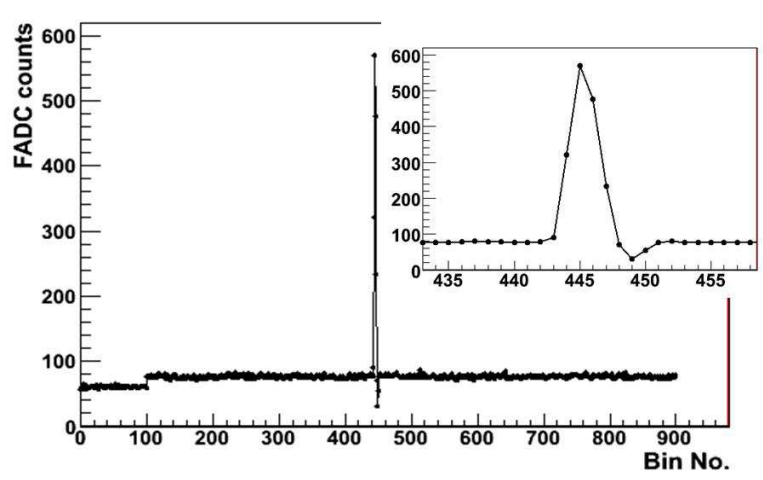

Figure 5: A Cherenkov signal with sky background; the first 100 bins are low gain background and the remaining 800 bins are high gain background and the Cherenkov signal. The Cherenkov signal is in the current frame (from the 300th bin to the 600th bin).

the stretcher. The AD8039 has a sufficiently high speed (350 MHz), low power dissipation, low cost, low noise, low distortion, and a nonlinearity less than $1 \%$ in a range of the input from $0.5 \mathrm{mV}$ to $800 \mathrm{mV}$, which nearly fits the entire range of the PMT signals.

To maintain good linearity over a wide dynamic range of 3.5 orders of magnitude in charge, a dual gain system is designed. Signals coming out of the AD8039 are split into two channels and are then separately amplified by two AD8138. With such a design, considerable flexibility in obtaining different resolutions of the charge measurements for pulses with different pulse heights is achievable by choosing the range covered by the high gain channel. In this paper, a ratio of the gains of 1:8 is selected as a result of the optimization between the dynamic range and the resolution in the charge measurement. The nonlinearity of AD8138 is less than $2 \%$ at both gains of 1 and 8 in the entire range of the input signals.

Another excellent feature of the AD8138, the conversion between a single-ended input and differential output for instance, makes it the best choice for an amplifier. Superposing an offset to the output of the AD8138 as a pedestal of the signal before feeding into 336 the positively-polarized FADC is also a convenient ap- ${ }_{337}$ proach. To manage possible undershoot of the pulses ${ }_{3 з 8}$ (see Fig 5), a pedestal is preset higher than the undershoot.

\subsubsection{Digitization Board}

Each DB has 8 pixel channels, which includes $16{ }_{343}$ FADC modules managed by two field programmable ${ }_{344}$ gate array (FPGA) modules. The 10-bit FADCs, 345 AD9215, has a good cost-performance ratio with a lin- 346

\subsubsection{First Level Trigger in a Single Channel}

The FLT is formed in the FPGA located on the DBs provided that the signal-to-noise ratio in a window is greater than a given threshold (e.g., 4 as a typical value). The width of the window is predetermined for corresponding observation modes (e.g., 8 bins for the Cherenkov light signals). Running over the entire frame of 300 cycles once in a bin, 293 sums of FADC counts in the windows (denoted as WINSUM) are produced. The average of WINSUM is calculated using these WINSUMs avoiding the maximal WINSUM and 


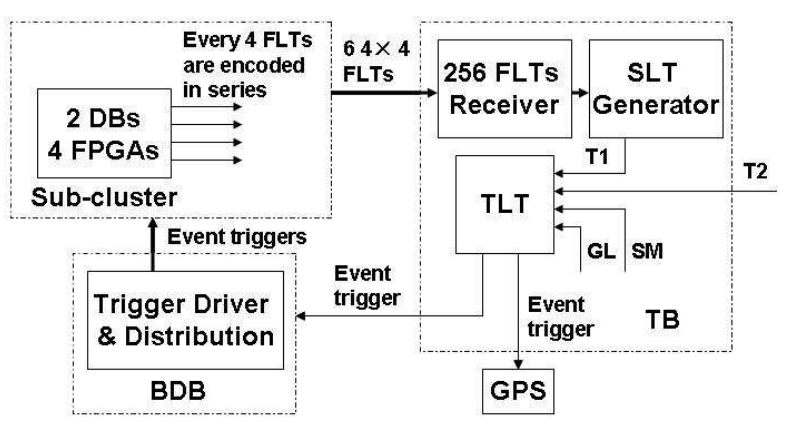

Figure 6: Three-level trigger system. SM is stereo or mono enable signal, GL is global or local enable signal, T1 is the telescope trigger from telescope No. 1 and T2 is from telescope No. 2. See detailed explanation of the diagram in the text.
18 WINSUMs on each side of the maximum. The stan- 384 dard deviation $(\sigma)$ of the WINSUM around the aver- 385 age serves as a measure of the background fluctuation. ${ }^{386}$ If the maximum of WINSUMs exceeds the average by ${ }^{387}$ $n \sigma$, the FLT is formed, where $n$ is preselected from a 388 list of $\sqrt{12}, \sqrt{16}, \sqrt{24}, \sqrt{32}$ and $\sqrt{40}$ before starting 389 the run. All trigger signals from 64 FPGAs are encoded 390 into bitmaps and transmitted to the Trigger Board (TB) 391 in parallel (see Fig 6).

The frame size of 300 bins is selected for a full scale ${ }^{393}$ array with more than 24 telescopes covering an area of 394 $1 \mathrm{~km}^{2}$, over which a highly inclined air shower takes 395 several microseconds to cross in the fluorescence light 396 observational mode. Such a frame is large enough to 397 fully contain the entire shower; it is also large enough 398 for Cherenkov light. All Cherenkov photons arrive at 399 the telescopes at almost the same time (few ns). There- 400 fore, an air shower signal appears only within 5 or 6401 bins after shaping and the night sky background is mea- 402 sured in the rest of the frame (see Fig.5). Such contin- 403 uous measuring of the sky background is highly useful not only in estimating the signal-to-noise ratio but also ${ }_{404}$ in monitoring the transparency of the atmosphere using well-known bright stars in the field of view.

\subsubsection{Second level trigger for a single telescope}

A trigger for a telescope registered by an air shower 409 (denoted as second level trigger or SLT) is formed when 410 a specific pattern of triggered PMTs corresponding to 411 a possible air shower is found in the camera of a tele- 412 scope. A pattern recognition technique, developed in 413 the Pierre Auger experiment [11] that is operated in a 414 FPGA located on the TB, is applied. Numerous pat- 415 terns, such as a fully filled circle formed with one hexag- 416 onal pixel surrounded by six others, or a straight line 417 formed by six aligned pixels, are pre-loaded into the 418
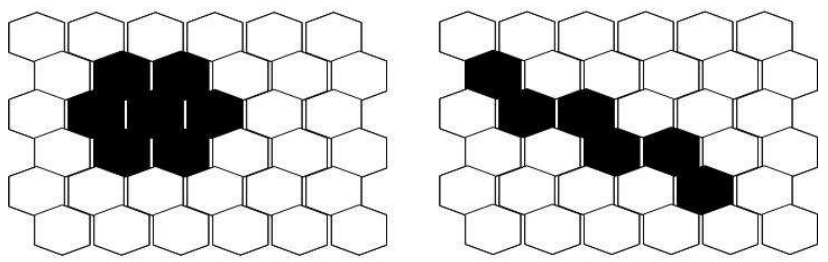

Figure 7: Two typical patterns in the second level trigger.

FPGA as a look-up table. Once all 256 FLT signals are collected, the FPGA matches all the pre-stored patterns with the observed one within a $6 \times 6$ matrix of pixels. Moreover, it keeps such a box running throughout the entire PMT camera with a step of one row or one column. The SLT is formed as long as any one of the prestored patterns is matched. According to the simulation for air showers, mainly two types of patterns exist. Fluorescence light images of air showers seen from several kilometers away tend to form line-shaped patterns on the camera, whereas Cherenkov light images of showers hitting the telescope head-on tend to form round-shaped patterns, as shown in Fig.7. There are 16 round-shaped patterns and 729 line-shaped patterns in a $6 \times 6$ box.

To speed up the formation of SLT, the pattern comparisons in the box are conducted in parallel. All 121 bitmaps of the boxes are generated by sliding the box are done in parallel. All 121 bitmaps of the boxes are generated by sliding the box throughout the camera. A pattern comparison algorithm is then carried out among the 121 bitmaps. The telescope trigger (SLT) is formed in 123 clock cycles, i.e., $2.46 \mu$ s.

\subsubsection{Third level trigger for entire array of telescopes}

An event trigger for the entire array of telescopes (denoted as the third level or TLT) is generated using one of the FPGAs used for SLT. For the two prototype telescopes, only two modes, namely, stereoscopic and monocular observation of showers, exists if they are configured in such a way that the two telescopes have a maximal overlap of the FOV. In stereoscopic mode, the two telescopes are required to simultaneously observe a shower. In monocular mode, each of the two telescopes can trigger the entire site. The operational mode should be selected at the beginning of a run by assigning two controlling parameters, SM and GL, as marked in Fig6.

The two telescopes communicate with each other through a two $80 \mathrm{~m}$ coaxial cables by sending telescope 
triggers (SLT) out and receiving the event trigger (TLT). 469 Such a trigger function is also useful for receiving an 470 external trigger and delivering a trigger to other co-sited 471 detectors. This makes the telescope an open and modu- 472 larized system.

\subsection{DAQ system}

The philosophy of the design of the Cherenkov tele- 476 scope DAQ is integrated and compact so that the en- 477 tire DAQ can be arranged on the backboard of the focal 478 plane camera. It allows for the maximal mobility of the 479 telescope for flexibility in switching between configura- 480 tions. Because of the limited space, a low power con- ${ }_{481}$ suming and compact-embedded online computer based ${ }_{482}$ on an ARM processor, industrial standard PC104 bus, ${ }_{483}$ and flash disk are selected as the backbone of the DAQ ${ }_{484}$ system, eliminating the need for moving parts such as ${ }_{485}$ the CPU fan and hard drives. All of the components are ${ }_{486}$ integrated on a bus driver board, which bridges the data ${ }_{487}$ storage disk and the $32 \mathrm{DBs}$ that are connected by flat ${ }_{488}$ cables. Because the event rate is not extremely high, ${ }_{489}$ i.e., around $1 \mathrm{~Hz}$, a band width of $14 \mathrm{MHz}$ for a PC104 490 bus is sufficient for transferring every bit of the $6 \mu$ s long 491 wave forms to the disk for all 256 channels. It is also ex- 492 tremely advantageous for system debugging. Each telescope is an independent detector with a complete DAQ system on board. The communication with the rest of ${ }^{493}$ the experiment is through a 10/100 Mbps Ethernet.

Each telescope has its own independent DAQ, devel- ${ }^{494}$ oped using $\mathrm{C}++$ language under Linux. Operating in 495 a polling mode instead of an interrupt mode, the DAQ 496 checks the interface status regularly for whether the data 497 are ready or not. If so, the data in the buffer are read 498 and immediately stored in the disk. Before a night shift 499 ends, the data in the hard disk are moved to the Institute 500 of High Energy Physics (IHEP) in Beijing for further 501 analysis.

\subsection{Power supply, slow control, and status monitoring 504}

The two power supplies with $+7 \mathrm{~V}$ (maximum current 505 $80 \mathrm{~A}$ ) and $-7 \mathrm{~V}$ (maximum current $20 \mathrm{~A}$ ) are installed in 506 the container. Regulators are used at each $\mathrm{AB}$ and $\mathrm{DB} 507$ to further stabilize the voltage. An adjustable HV power 508 supply with a maximal output of $-2000 \mathrm{~V}$ and $100 \mathrm{~mA}{ }_{509}$ is used for each telescope.

One of the difficulties at high altitudes is the heat dis- 511 sipation of the power supplies and electronics enclosed 512 in a metal box. The total power consumption is about ${ }_{513}$ $50 \mathrm{~A}$ at $+7 \mathrm{~V}$ and $16 \mathrm{~A}$ at $-7 \mathrm{~V}$. A forced cooling sys- 514 tem is necessary in the prototype experiment to improve 515 the heat dissipation of the entire camera and the power 516 supplies.
The entire telescope system is powered by an uninterrupted power supply with a sufficient battery backup. It protects the telescopes from damage when a blackout occurs.

The detector is designed to work in remote control mode, including the opening and closing of the doors, turning the power supplies on/off, enabling/disabling the high voltage (HV) power supply and low voltage (LV) power supplies, and switching the UV LEDs on/off for detector calibration. All kinds of controls are realized using the on-board computer through a COM port. Carefully monitoring the status of each telescope is necessary, including the door status, voltages, and temperatures at different places (e.g., enclosed areas inside the camera, backboard of the PMT camera, and inside the housing of the UV LED). All parameters are measured and recorded through an 8-channel 12-bit analogto-digital-converter on the board of the embedded computer, TS7200.

All of the controls are performed through a user interface running at the embedded computer connected through Ethernet from an operational center, $3000 \mathrm{~km}$ away in IHEP in Beijing. A small portion of the data can be copied to display the event during the operation.

\section{Calibration}

\subsection{Method of calibration}

An accurate shower reconstruction requires a converting factor from a pulse area in terms of FADC counts to the number of photons for each pixel. Therefore, the absolute calibration of the detector response is essential. To achieve this objective, having an accurate knowledge about PMT cathode effective areas, cathode quantum efficiency, PMT gains, amplifier gains, and digital converting factors, is necessary. Measuring all these effects item by item is difficult. In this paper, a method similar to the HiRes experiment [14] and Pierre Auger experiment [12] calibration procedure, which considers the entire effect, is applied to measure the overall response of each pixel. The procedure is discussed in the paragraphs that follow.

Being mounted at the center of the mirror, a UV-LED $(375 \mathrm{~nm})$ light source with a diffuser is used for the calibration of PMTs in the camera by beaming nearly uniform light to every pixel. The LED light density on the camera surface is calibrated using a pre-calibrated probe detector located beside the camera. The calibration of all pixels in the cameras is performed twice a day, i.e., before and after the daily operation. The crucial part is the measurement of the absolute number of UV photons 
at each PMT cathode from a pulse emitted by the LED, which is performed in two steps.

First, we move one PMT over all the places on the frame of the PMT camera to measure the uniformity of the LED light density on the camera surface. This has to be accomplished before the PMT camera is installed. The light density is a function of the polar angle, $\theta$, in a form of $\cos ^{4} \theta$, where $\theta$ is the angle between the connection from the LED to the PMT location and the perpendicular connection from the LED to the PMT camera surface. The variation from the center to the corner of the PMT camera is within $7 \%$.

In the second step, the light density is measured using a pre-calibrated probe consisting of two XP3062 PMTs with the same FEE and DAQ as the two telescopes. The only difference is that the two PMTs in the probe are operated at a very high gain so that the single photoelectron can be measured. Therefore, the gain of the probe, $G^{\text {probe }}$, can be calibrated at any time. Then, the absolute calibration of the probe is performed at the HiRes lab at the University of Utah, USA, by comparing the response of a hybrid photo diode (HPD) pre-calibrated at NIST [18] to the same light source. The probe and HPD are located side by side in front of a UV-LED at $355 \mathrm{~nm}$. Using the HPD, we measure light density $I_{U}$ (number of photons per square millimeter) from the LED. We also measure pulse area $F_{U}^{\text {probe }}$ using the probe simultaneously. At the operational site in Tibet, the light density from the LED mounted on the center of the mirror is calibrated to be $I_{T}=I_{U} \frac{F_{T}^{\text {probe }}}{F_{U}^{\text {probe }}} \frac{G_{U}^{\text {probe }}}{G_{T}^{\text {probe }}}$, where $U$ and $T$ stand for Utah and Tibet, respectively.

Applying the knowledge obtained from the two steps, 576 we have determined both the light density in front of 577 the cathode and the pulse area in terms of the FADC 578 counts for each PMT in the camera. Finally, the over- 579 all converting factor for a pixel between the number of 580 photons reached to the surface of the camera and cor- 581 responding pulse area measured by the FADC counts 582 behind the pixel is

$$
C_{375}^{\text {camera }}=\frac{F_{T}^{\text {camera }}}{I_{T} A_{P M T}} \frac{\cos ^{4} \theta^{\text {probe }}}{\cos ^{4} \theta^{\text {camera }}},
$$

where $A_{P M T}$ is the geometric area of the PMT cathode, ${ }^{586}$ and $C_{375}^{\text {camera }}$ is the calibration constant for a pixel. The ${ }_{588}$ subscript 375 indicates that the calibration is done using UV light at $375 \mathrm{~nm}$. The unit of the calibration constant is FADC counts per photon. $\theta^{\text {probe }}$ and $\theta^{\text {camera }}$ represent 589 the angular locations of the probe and the pixel in the camera, respectively. A further correction according to ${ }^{590}$ the wavelength dependence of the quantum efficiency 591 of PMTs is applied in the operation for the cosmic ray 592 observation.

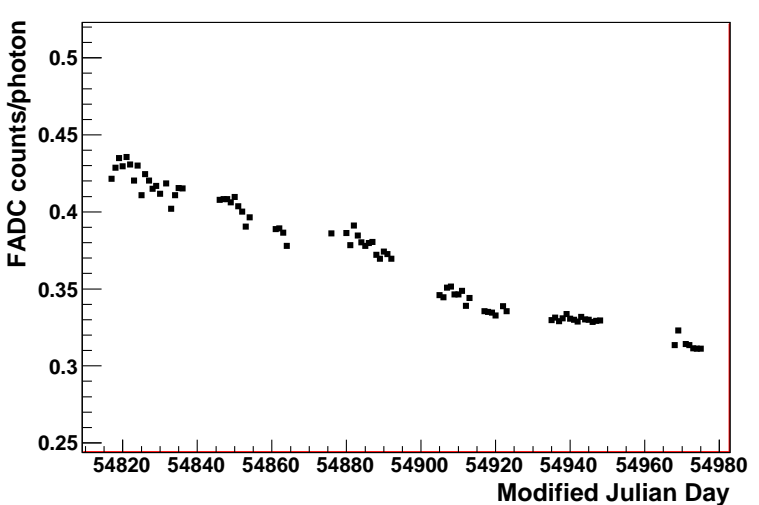

Figure 8: Absolute gain of one telescope from Dec. 2008 to May 2009 , as a function of time.

\subsection{Result of the calibration}

The probe was calibrated at the HiRes lab. The calibration results are shown in table 1, the gain of the probe is $85.7 \pm 0.3$ FADC count per SPE. The second column shows the lower LED photon density and the third column shows the higher LED photon density that coincide with one another.

Table 1: The calibration results of the probe.

\begin{tabular}{|c|c|c|}
\hline$I_{U}\left(\right.$ photons $\left./ \mathrm{mm}^{2}\right)$ & $0.359 \pm 4.8 \%$ & $0.538 \pm 4.8 \%$ \\
\hline$F_{U}^{\text {probe }}(\mathrm{FADC}$ count $)$ & $8959 \pm 4$ & $13650 \pm 5$ \\
\hline
\end{tabular}

The corresponding calibration constant for one of the telescopes is presented in Fig 8 An average of the calibration constants of all pixels in the camera is plotted with respect to time from December 2008 to May 2009. As mentioned above, the calibration constant is monitored every observational day. The systematic uncertainty of the calibration constant is estimated to be $7 \%$. The downtrend of the constant shown in the figure indicates obvious decreases of gains in all newly produced of PMTs. The transmission of the glass window and reflectivity of the mirrors are not take into account in the above calibration. These two effects will be monitored in so called end-to-end calibration using nitrogen laser in future.

\section{Performances and Results from Test Run}

\subsection{Test Run Information}

The two telescopes began recording cosmic ray data in August 2008. Furthermore, both monocular and stereoscopic modes have been tested. About 500,000 
coincidental events with the ARGO-YBJ experiment in stereoscopic mode and 700,000 coincidence events in monocular mode have been collected up to January 2010. The average trigger rate is about $0.5 \mathrm{~Hz}$ in stereoscopic mode and $0.7 \mathrm{~Hz}$ in monocular mode.

\subsection{Off-line Coincidence with the ARGO-YBJ Experi- ment}

All WFCTA telescopes and the ARGO-YBJ experiment recorded the cosmic ray arrival time based on a GPS. A time window of $8 \mu$ s containing a Cherenkov event is searched for coincidence with the ARGO-YBJ event stream, which is about $4 \mathrm{kHz}$. A difference between the recorded event time by the two experiments for a matched event is typically less than $100 \mathrm{~ns}$ (Fig 9).

For a coincidence event, the shower geometry is measured using the ARGO-YBJ detector. The distribution of shower arrival directions is shown in a 2-dimensional map, i.e., zenith angles versus azimuth angle, as in Fig 10 Approximately $85 \%$ of coincident events occur inside the FOV of the Cherenkov telescopes marked by the trapezium. The rest of the $15 \%$ of events have their images partially seen by the telescopes and sufficiently trigger the telescopes. The shower core distribution is shown in Fig 11 The ARGO-YBJ experiment array and the two Cherenkov telescopes are marked as the rectangle and two dots in the figure, respectively. For events that have the reconstructed cores inside the ARGO-YBJ array, the shower parameters, such as the number of hits on the carpet detector $\left(N_{h i t}\right)$ and shower geometry, are well measured. A distribution of $N_{h i t}$ of these events is plotted in Fig 12. According to the number of hits as a function of the primary energy of proton from ARGOYBJ detector [19], the mode energy of protons is about $40 \mathrm{TeV}$. This estimates the threshold of two Cherenkov telescopes in stereoscopic mode.

In Fig 13, two Cherenkov images are shown in the 644 event display. The top image is caused by an event at ${ }_{645}$ a $10 \mathrm{~m} R_{p}$ (the impact parameter of the shower to the 646 telescope) and the bottom image stems from an event at ${ }_{647}$ a $173 \mathrm{~m} R_{p}$. The image is clearly more elongated for the ${ }_{648}$ farther event. Using the well-defined image parameters 649 created by Hillas [21], length and width, this effect is 650 more quantitatively presented in Fig 14 The widths of 651 the images seem to be no longer shrinking once show- 652 ers are sufficiently far from the telescopes (e.g., farther ${ }_{653}$ than $100 \mathrm{~m}$ ). The showers essentially resemble linear 654 patterns.

\subsection{Electronic noisy and sky background}

For a triggering system completely based on the 658 signal-to-noise ratio, such as the WFCTA telescopes, 659

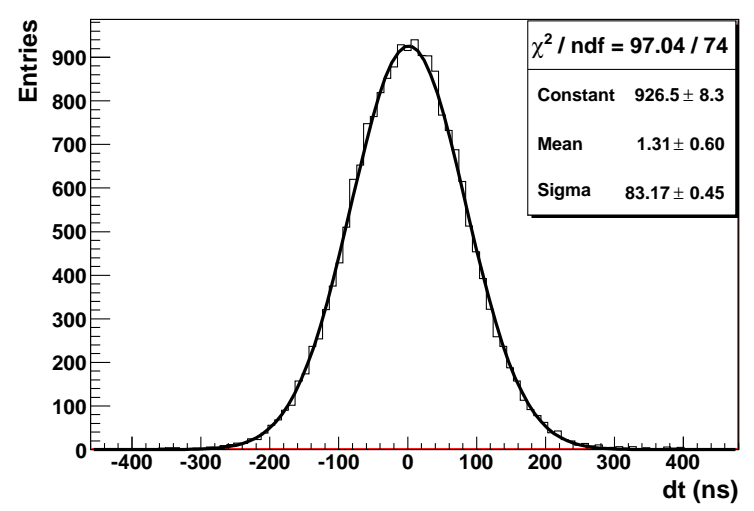

Figure 9: The difference time between WFCTA and ARGO in coincidence event.

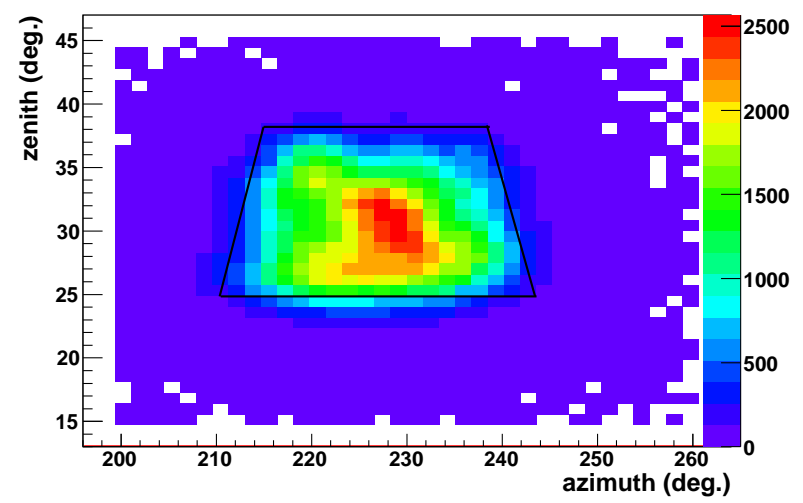

Figure 10: Distribution of coincidence events are seen by ARGO and Cherenkov telescopes simultaneously over zenith and azimuth angle. About $85 \%$ of coincidence events are located in the Cherenkov telescope FOV (trapezium). The image of the rest of the $15 \%$ of coincidence events is partially in the Cherenkov telescope FOV.

the sensitivity is constrained by the noise level. For a most optimized system, the electronic noise must be negligible compared with the night sky background. Both of them are measured during the operation as the door is closed and opened, respectively.

The sources of electronic noise include thermal noises in the PMTs, noises from the HV power supply and LV power supplies, noises of amplifiers, and finally from the counting error of FADCs. The PMT Photonis XP3062/FL has a very low dark current; therefore, the thermal noise level is sufficiently low so that it can be ignored compared with the other sources of electronic noises. The HV power supply has a ripple less than $0.02 \%$ in terms of RMS of rated voltage. This contributes a variation within $0.12 \%$ in terms of the gain of the PMTs. Voltage regulators are used to block pos- 


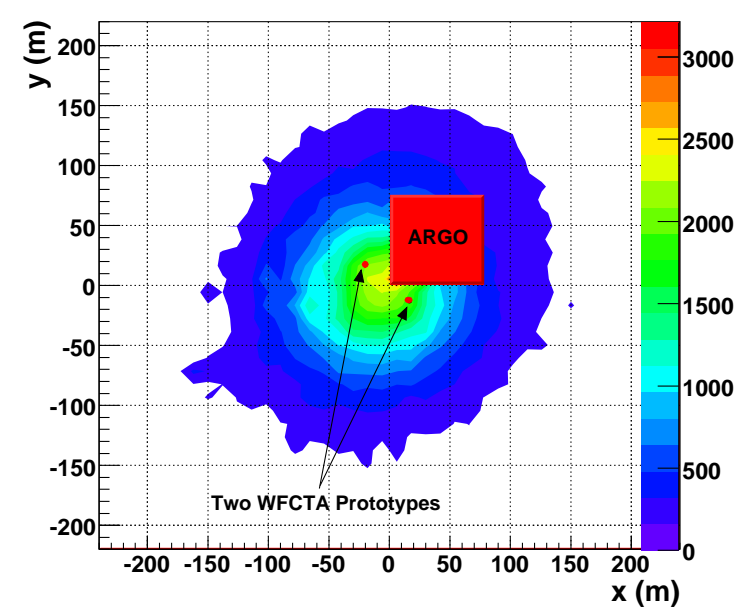

Figure 11: Distribution of shower core. Two Cherenkov telescopes (two dots) and the ARGO-YBJ experiment array (rectangle) are also marked in the figure.

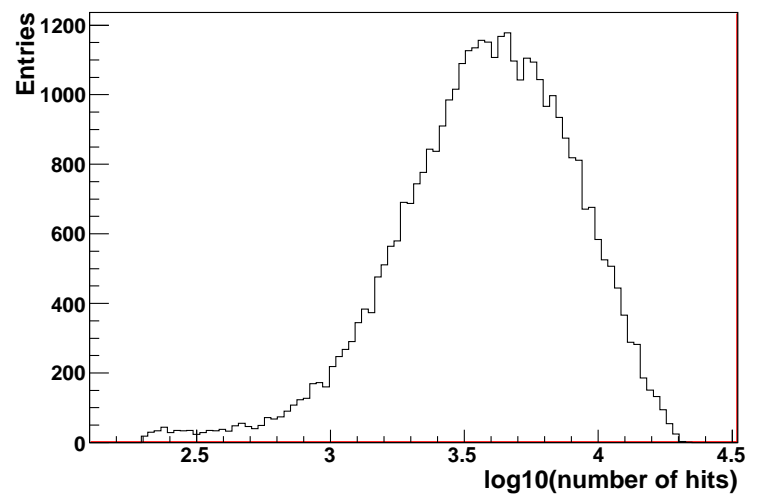

Figure 12: Distribution of ARGO nhit of these coincidence events, whose reconstructed core is located in the ARGO-YBJ cluster array. sible noises from the LV power supplies. All amplifiers, AD8138 and AD8039, are selected to generate a very low noise level. The noise from the FADC is about 0.5 FADC counts per tube. Taking into account all additional sources such as the distributing capacity on boards and connectors, the total electronic noise, including PMT and high voltage power supply, is typically about 1.0 FADC counts per tube. It is measured during the calibration with the LED (Fig $\sqrt[15]{ }$ ) as long as 68 the signal is avoided.

The night sky background is measured similarly as 600 a shower trigger is formed when the doors are open 687 (Fig 5). The fluctuation in the night sky back-ground is 688 typically more than 2.2 photon electrons per $20 \mathrm{~ns}$ per 689 tube on a clear moonless night. This suggests a much 690
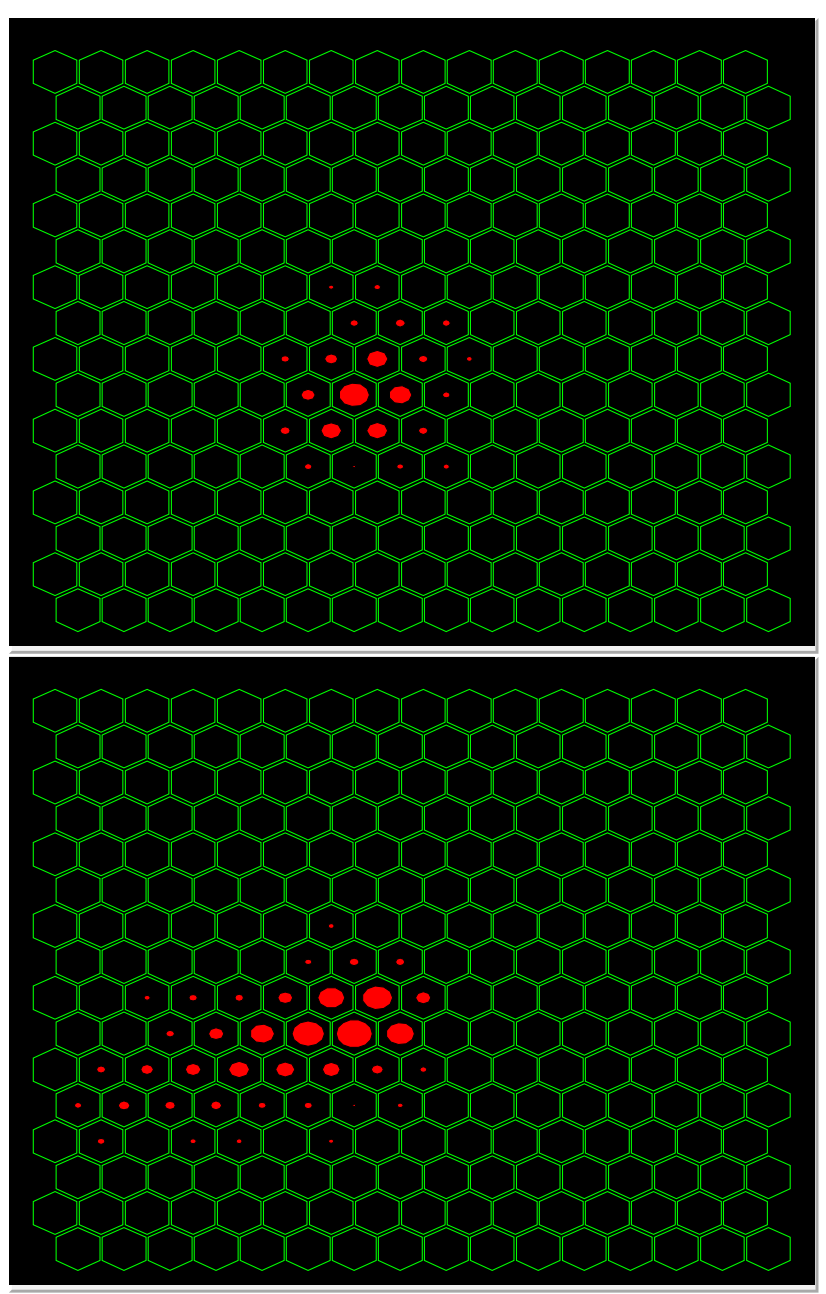

Figure 13: Two Cherenkov events; the Rp of the tope event is $10 \mathrm{~m}$ and that of the bottom event is $173 \mathrm{~m}$; the image of the bottom event has a longer tail than the top event

stronger noise than electronic noises. One of the important sources of the sky background is light from the stars (Fig 16), which shows the sky background at night, in which each pulse denotes stars passing though the FOV. A bright individual star can be traced when it passes through the telescopes. Stars provide numerous stable point-like sources that typically sweep across a tube in about 4 minutes. Those ideal point-like sources can be used for multiple calibration purposes. For instance, well-known bright stars can be used as light houses to establish the pointing direction of the telescope itself, with accuracy proved to be better than $0.05^{\circ}[20]$. A bright star can be seen as a very stable point-like source at infinity, which is a perfect tool for measuring the spot size produced by the optical system. The background of a tube signal steadily increases when the light spot 


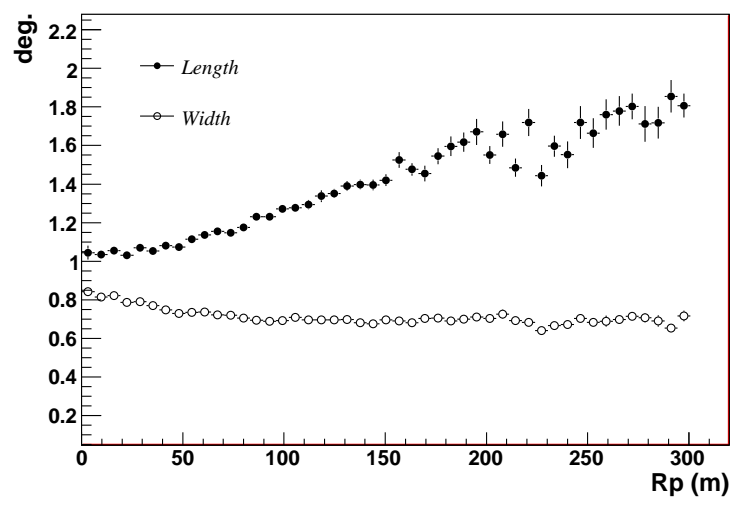

Figure 14: Length (filled circles) and width (open circles) as a function of Rp.

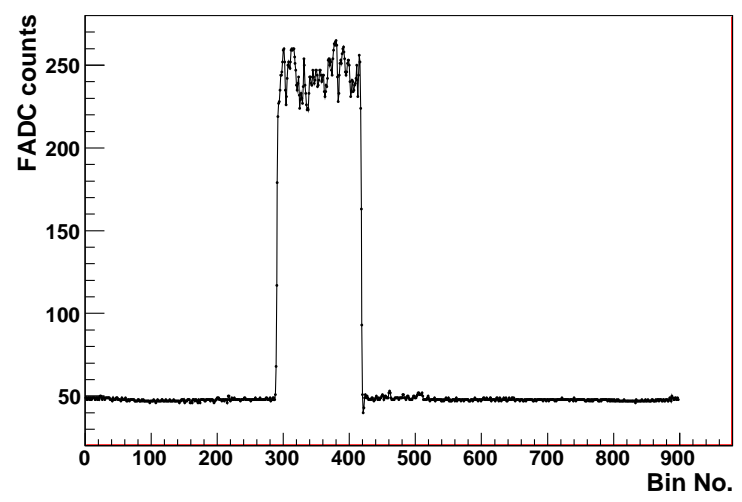

Figure 15: LED calibration for door closed. The low noise level of 1 LSB of the complete electronics is to be seen before and after the LED pulse. moves into the FOV of the tube, and steadily decreases when it moves away from the FOV, forming a light profile. The light profile can be fitted using a Gaussian function, whose sigma denotes the spot size. The spot size grows larger when the star moves away from the center of camera (Fig 17).

\section{Summary}

The telescopes were successfully run at YBJ from ${ }^{71}$ August 2008 up to July 2009. Millions of coincidence ${ }^{712}$ events with the ARGO-YBJ experiment have been collected. The performance of the telescopes was studied using these events. The trigger rate is about $0.5 \mathrm{~Hz}$ in 714 stereo mode. Moreover, the mode energy of the tele- 715 scope is $40 \mathrm{TeV}$ when a pure proton composition is as- 716 sumed.

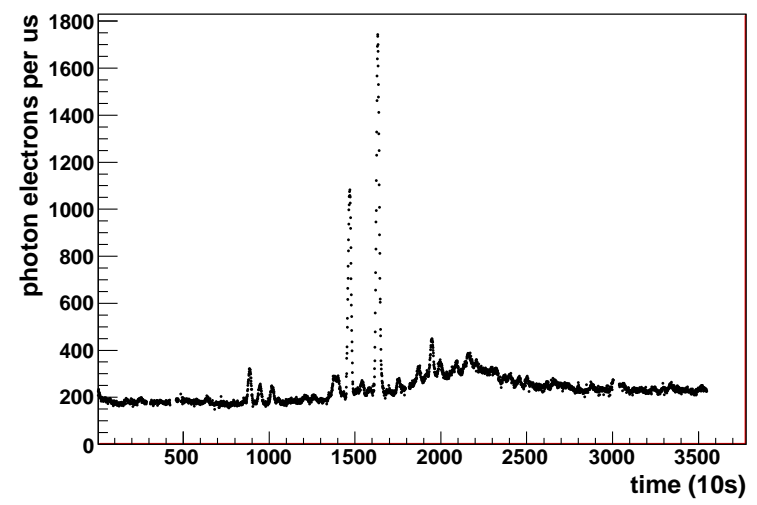

Figure 16: Sky background is monitored by a PMT in a clear moonless night; each pulse corresponds to a star passing through the FOV of the telescope.

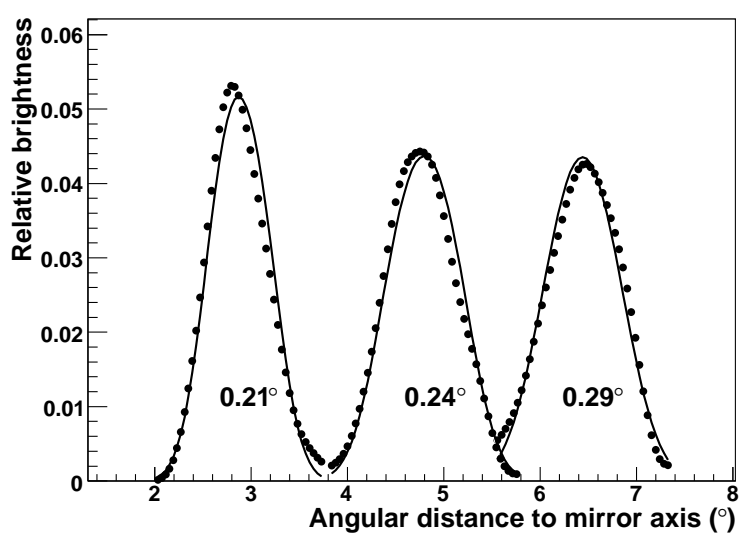

Figure 17: A star moves away from the center of the camera, and its light profile recorded by PMT is drawn in filled circles. The light profile described by a Gaussian function is drawn in a solid line. The sigma of Gaussian denotes that the spot size is also marked.

The features of the two WFCTA prototype telescopes are summarized as follows:

- a $4.7 \mathrm{~m}^{2}$ spherical mirror,

- a $16 \times 16$ PMT array covers an FOV of $14^{\circ} \times 16^{\circ}$ with $1^{\circ}$ pixels,

- dual gain system for a dynamic range to 3.5 orders of magnitude,

- DC coupling and modulized design for electronics,

- three-level online trigger logic: single channel trigger based on $\mathrm{S} / \mathrm{N}$ ratio, telescope trigger based on pattern recognition, and event trigger for stereoscopic observation, 
- maximized mobility and the telescope can be up- 772 lifted from $0^{\circ}$ to $60^{\circ}$ in elevation.

The absolute gains of the telescopes are calibrated using calibrated LEDs mounted at the centers of the mirrors. The systematic uncertainty of the calibration constant is about $7 \%$. The pixel gains are monitored on a daily basis.

\section{Acknowledgements}

This work is supported by the Chinese Academy of Sciences (0529110S13) and the Key Laboratory of Particle Astrophysics, Institute of High Energy Physics, CAS. The Knowledge Innovation Fund (H85451D0U2) of IHEP, China and the project Y0113G005C of NSFC also provide support to this study.

We are very grateful to the ARGO-YBJ Collaboration for authorizing us to use the data of the ARGO-YBJ experiment.

We also acknowledge the essential support of H. M. Zhang, W.Y. Chen, G. Yang, X.F. Yuan, C.Y. Zhao in the installation, debugging, and maintenance of the detector.

\section{References}

[1] V.I. Zatsepin et al., Nucl. Instr. and Meth. A 524 (2004) 196 195-207

[2] H.S. Ahn et al. Nucl., Instr. and Meth. A 579 (2007) 1034-1053

[3] Zhen Cao et al., A Future Project at Tibet: The Large High Altitude Air Shower Observatory (LHAASO), Chinese Physics C 2010, 34 (02): 249-252

[4] Huihai He et al., LHAASO Project: detector design and prototype, 31st ICRC, LODZ,(2009).

[5] Z. Cao et al., j. Phys. G: Nucl. Part. Phys. 31 (2005) 571

[6] He HuiHai et al., Proc. of 30th ICRC, Vol. 5 (2007) 949

[7] G. Aielli et al., Nuclear Physics B (Proc. Suppl.) 166 (2007) 96-102.

[8] J. Abraham et al., The Fluorescence Detector of the Pierre Auger Observatory, Nucl. Instr. and Meth. A620 (2010) 227-251

[9] J. Abraham et al., Measurement of the energy spectrum of cosmic rays above $10^{18} \mathrm{eV}$ using the Pierre Auger Observatory, Phys. Letter B685 (2010) 239-246

[10] T.Nonaka et al., The present status of the Telescope Array experiment, Nuclear Physics B (Proc. Suppl.) 190 (2009) 26-31

[11] J. Abraham et al., Nucl. Instr. and Meth. A 523 (2004) 50-95

[12] P. Bauleo et al., 29th International Cosmic Ray Conference Pune (2005) $8,5.8$

[13] John H. Boyer et al., Nucl. Instr. and Meth. A 482 (2002) 457 474

[14] D. Bird et al., Nucl. Instr. and Meth. A 349 (1994) 592-599

[15] T. Abu-Zayyad et al., 2001 ApJ 557686

[16] S. P. Swordy, D. B. Kieda, arXiv:astro-ph/9909381 v1

[17] G.Xiao et al., 29th International Cosmic Ray Conference Pune (2005) 8, 21-24.

18] L.P. Perera, Calibration of the Roving Xenon Flasher with a Hybrid Photodetector internal HiRes report, 2003, http : //www.cosmic - ray.org/papers/Lalith_Hpd.pdf
[19] GUO Yi-Qing et al, Chinese Physics C, 2010, 34 (5): 555-559

[20] Ma Ling-Ling et al, Geometry and optics calibration of WFCTA prototype telescopes using star light, Chinese Physics C, proceeding.

[21] Hillas A. 1985, in Proc. 19nd I.C.R.C. (La Jolla), Vol. 3, p. 445. 